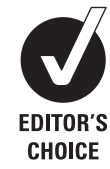

CHOICE

\title{
The investigation and management of hirsutism
}

\author{
Stephen Franks
}

Professor of Reproductive Endocrinology, Institute of Reproductive \& Developmental Biology, Imperial College London, London, UK

\section{Correspondence to} Professor Stephen Franks, Institute of Reproductive \& Developmental Biology, Imperial College London, Hammersmith Hospital, London W12 0NN, UK; s.franks@imperial.ac.uk

Received 14 July 2011 Accepted 16 April 2012

\begin{abstract}
Excess male-pattern body hair in women is a very common and psychologically damaging condition. Although its cause is usually a chronic and benign disorder (most commonly polycystic ovary syndrome) it may rarely be an indication of a more serious endocrine disease such as Cushing syndrome or an androgen-secreting tumour. Investigations do not usually need to be extensive, but effective management is important, irrespective of cause, for what can be a debilitating symptom. Specific treatment of any underlying disease is important but in most cases treatment is empirical; it may simply involve physical hair removal, ideally by electrolysis or laser treatment. However, endocrine therapy to suppress androgen production and/or action is desirable in many, if not most, cases.
\end{abstract}

\section{Introduction}

Hirsutism in women is defined as excessive terminal hair in a male-pattern distribution. ${ }^{12}$ Although there are objective methods of assessing the extent of hirsutism, the perception and impact of excess body hair in an individual woman depends not only on its extent and severity but also on social and cultural influences. ${ }^{3}$ It is clear that the cutaneous expression of androgen excess is profoundly affected by ethnic origin, so that hirsutism is a much more prevalent symptom of hyperandrogenism in women from the Indian subcontinent and from Mediterranean countries than it is in those of northern European or east Asian origin.

Clinical assessment of hirsutism should include taking a careful history regarding duration and severity of hair growth as well as its impact on daily life. ${ }^{3}$ It is important to ask how much time the woman spends in removing body hair, and by which method. Quality of life studies have indicated that severe hirsutism has a serious adverse effect on social interactions and that affected women have a high incidence of depressive symptoms. ${ }^{4-6}$ The Ferriman-Gallwey chart provides a simple, semi-quantitative scoring system for assessment of distribution and severity of excess body hair and is used in many endocrine and dermatological clinics. ${ }^{13}$

\section{Key message points \\ Hirsutism is very common; the causes are usually benign but the psychological impact can be severe. \\ - Few investigations are needed in most cases of hirsutism. \\ - Effective treatment usually requires a combination of physical hair removal and endocrine treatment.}

\section{Androgen production, transport and metabolism in women}

In normal premenopausal women, the ovaries and the adrenals contribute equally to circulating testosterone concentrations. $^{78}$ About 50\% of serum testosterone results from direct secretion by ovaries and adrenals and the remainder is derived from metabolism of androstendione, a more prevalent but weaker androgen, in peripheral tissues such as skin and fat. Dehydroepiandrosterone (DHEA) and dehydroepiandrosterone sulphate (DHEAS) - quantitatively the most abundant circulating androgen - are also weak androgens that are predominantly of adrenal origin. The ovary produces a small amount of DHEA, but DHEAS is a reliable marker of adrenal androgen secretion, especially in conditions of androgen excess. The most prevalent causes of androgen excess in premenopausal women are ovarian in origin (see later) but adrenal androgen excess, while less common, is nevertheless an important cause of hirsutism.

Most circulating testosterone is bound to protein, either sex hormone-binding globulin (SHBG) (around 80\%) or albumin (about 19\%). Only about 1\% of testosterone is therefore 'free' in the circulation and it is this free fraction that is considered to be the bioavailable form and therefore active in target tissues. ${ }^{9}$ There remains some controversy as to whether protein-bound testosterone is also biologically available under certain conditions, 
but it is clear that changes in the serum concentrations of SHBG have an effect on the bioactivity of testosterone.

Of course, in the context of hirsutism the most important testosterone target tissue is the skin and much is known about the local metabolism of androgens. ${ }^{10}$ The hair follicle itself contains not only androgen receptors located in the dermal papilla, but also various steroidogenic enzymes that are involved in androgen production and metabolism ${ }^{11}$ including $3 \beta$-hydroxysteroid dehydrogenase (3ßHSD), 17ßHSD and, arguably the most important, $5 \alpha$-reductase, which converts testosterone to the more potent dihydrotestosterone. This hormone binds with high affinity to the androgen receptors in hair follicles. ${ }^{10}$

\section{Causes of hirsutism}

The causes of hirsutism are summarised in Table 1 . Polycystic ovary syndrome (PCOS) accounts for the vast majority of cases ${ }^{12-15}$ and it is important to realise that PCOS includes not only those women with the classic combination of oligo-amenorrhoea and hirsutism but also those who have regular cycles and hirsutism. ${ }^{13}$ Over $80 \%$ of women with hirsutism and regular cycles, who may previously have been regarded as having 'idiopathic hirsutism', ${ }^{12}{ }^{13}$ have polycystic ovaries and, as a group, have the associated features of hyperandrogenaemia and higher-than-normal serum concentrations of luteinising hormone (LH). Androgen-secreting tumours of the ovary are rare but their early recognition is important (see investigative tests below).

In women with PCOS, adiposity is an important factor in determining the prevalence and severity of hirsutism. Overweight and obese women with PCOS are more likely to be hirsute than those who are lean. ${ }^{16}{ }^{17}$ Women with PCOS who gain weight often notice an increase in extent and severity of hirsutism and, conversely, those who lose weight may experience an improvement in this symptom. ${ }^{18}{ }^{19}$ Hyperinsulinaemia is a feature of obesity, but circulating insulin levels are relatively higher in women with PCOS-related obesity. There are at least two mechanisms by which elevated circulating insulin

Table 1 Causes of hirsutism
Ovarian
- Polycystic ovary syndrome (PCOS) $(>80 \%)$
- Hyperthecosis
- Ovarian tumours (sex cord stromal tumours; Sertoli-Leydig cell
tumours; adrenal-like tumours of the ovary) $(<1 \%)$
Adrenal
- Congenital adrenal hyperplasia [classical $1 \%$; non-classical (late-onset) $3 \%$ ]
- Cushing syndrome $(<1 \%)$
- Adrenal tumours (adenoma; carcinoma) $(<1 \%)$
Idiopathic
- With raised androgens $(5 \%)$
- Without raised androgens $(7 \%)$

concentrations, the result of insulin resistance in muscle and adipose tissue, affect androgen action. The first is a direct effect of insulin on androgen production by the ovary as insulin itself stimulates ovarian theca cells to overproduce androgens. ${ }^{20}$ The second effect is that the higher the serum insulin, the lower the circulating concentration of SHBG, and this leads to a higher free fraction (and therefore greater bioactivity) of circulating testosterone. Here, the mechanism is attributable to the ability of insulin to suppress hepatic production of SHBG. ${ }^{21}$

Ovarian hyperthecosis is an uncommon condition in which androgen-secreting theca-interstitial cells are not just confined to antral follicles, but are dispersed through the ovarian stroma. They may therefore continue to function after the menopause and indeed may secrete even more androgen in response to the postmenopausally elevated concentrations of LH.

The commonest adrenal cause of hirsutism is non-classical (late-onset) congenital adrenal hyperplasia $(\mathrm{CAH})$ due to deficiency of the 21-hydroxylase enzyme. ${ }^{22}$ The less common, classical (early-onset, salt-losing) form of $\mathrm{CAH}$ is usually diagnosed in infancy (or even in utero) and early treatment can prevent development of symptoms and signs of androgen excess. Late-onset $\mathrm{CAH}$ is difficult to distinguish clinically from $\mathrm{PCOS}^{22}$ and the differential diagnosis is further complicated by the fact that the majority of women with biochemically proven $\mathrm{CAH}$ also have polycystic ovaries on ultrasound examination. Other important adrenal causes include Cushing syndrome and adrenal tumours but these are rare, and important clues to diagnosis such as short duration, severe symptoms and highly elevated serum testosterone levels may be gleaned from history and initial investigations. Of course, there still remains a minority of women in whom no obvious cause of hirsutism can be found, but truly idiopathic cases now make up little more than $10 \%$ of the total.

\section{Investigation of hirsutism}

A guide to the investigation of hirsutism is provided in Table 2. It could be argued that no investigations are needed in women who have mild, long-standing hirsutism that is controllable with simple cosmetic treatment and who have regular menstrual cycles. ${ }^{2}$ They are likely to have polycystic ovaries or idiopathic hirsutism. However, the practice in our clinic is to perform a pelvic ultrasound scan and a serum testosterone measurement, mainly to be able to offer the patient a diagnosis. The main purpose of serum testosterone measurement in any woman with hirsutism is as a screening test to exclude more serious causes of androgen excess. Women with chronic hirsutism and regular cycles are likely to have normal or modestly elevated serum levels of testosterone. There are issues regarding the precision and specificity of commercially available immunoassays if testosterone levels are in the normal female range. ${ }^{23}$ For this reason many laboratories have 
Table 2 Investigation of hirsutism

\begin{tabular}{|c|c|}
\hline Diagnosis & Investigation/test \\
\hline Mild, chronic hirsutism, regular cycles & No tests? (or testosterone, pelvic ultrasonography) \\
\hline Moderate hirsutism with or without cycle disturbance & Testosterone, LH, FSH, ultrasonography \\
\hline Severe hirsutism, short history, testosterone $>5 \mathrm{nmol} / /^{\star}$ & $\begin{array}{l}\text { DHEAS, 17-OHP, dexamethasone suppression test, 24-hour urine free cortisol, } \\
\text { ovarian and/or adrenal imaging, fasting glucose/insulin }\end{array}$ \\
\hline
\end{tabular}

turned to tandem mass spectrometry assays, but it is a useful rule of thumb to state that a testosterone level within the normal range, by any method, is unlikely to be associated with serious pathology. It is higher levels that should give cause for concern and that indicate the need for further investigation. Routine measurement of SHBG and calculation of the free testosterone index (FTI) (testosterone/SHBG $\times 100$ ) are not indicated in the investigation of hirsutism. Although FTI values are more commonly abnormal than levels of total testosterone in hirsute women, they are of little extra value in clinical management.

If there is a moderate degree of hirsutism, particularly if associated with menstrual cycle abnormalities (oligomenorrhoea or amenorrhoea), PCOS is by far the most common cause and this largely clinical diagnosis should be supported by ultrasonography and endocrine tests: serum testosterone, $\mathrm{LH}$ and follicle-stimulating hormone in the first instance. The differential diagnosis includes non-classical CAH, but the value of testing specifically for 21-hydroxylase deficiency depends on the expected background prevalence of $\mathrm{CAH}$ in the local population and whether, if positive, management of hirsutism is likely to be any different from that in women with PCOS - it is usually very similar. The definitive tests for $\mathrm{CAH}$ are measurement of 17-hydroxyprogesterone (17-OHP) before and after stimulation with synthetic adrenocorticotropic hormone (ACTH) (a short Synacthen test) and genetic screening.

The need for more extensive tests arises in women who present with severe hirsutism, those with a short duration of worsening hirsutism or those whose serum testosterone is significantly elevated. An arbitrary guide is a level more than twice the upper limit of the normal female range for the laboratory. Androgen-secreting tumours of the ovary or adrenal should be suspected if the serum testosterone level is in the male range, $10 \mathrm{nmol} / \mathrm{l}$ or above. Occasional women with PCOS and severe insulin resistance can have testosterone levels in the male range. The clue to this diagnosis is the presence of acanthosis nigricans, which is a cutaneous marker of hyperinsulinism.

Measurement of DHEAS is a useful indicator of adrenal androgen excess. A 24-hour urine free cortisol measurement is a good screening test for Cushing syndrome, ${ }^{2}$ with high sensitivity (few false-negative results) but rather low specificity. Thus a positive test requires follow-up with more specific investigations for hypercortisolism, usually at a tertiary centre. Imaging of the ovaries and adrenals by ultrasound or magnetic resonance imaging is essential in cases where a tumour should only be suspected. Selective venous catheterisation is rarely required and should only be performed in endocrine centres with extensive experience of this technique. The use of other tests depends on clinical and biochemical pointers, for example, fasting insulin levels in women with PCOS, acanthosis nigricans and very high testosterone levels.

\section{Management of hirsutism}

Effective treatment of hirsutism requires the combined approach of physical methods of hair removal and the use of medication - principally, but not exclusively, hormonal therapy ${ }^{24}$ (Table 3). It is important to invest time in discussing the various methods of hair removal with the patient and to emphasise, for example, that shaving does not lead to faster or stronger regrowth of hair, as is commonly perceived. Ideally, a beautician with experience in the management of hirsutism would be available but, not surprisingly, few clinics or GP practices can easily draw on such expertise. It is often appropriate to seek the advice of a dermatologist, many of whom have a special interest in the management of hirsutism. A recommendation to a reliable local electrologist or to a laser clinic will help. In the UK, accredited electrologists can be found via the website of the British Institute \& Association of Electrolysis (http://www.electrolysis.co.uk). Laser hair removal is most effective for women with dark hair and light skin. Unfortunately such treatment, although best in long-term management, is rarely available within the National Health Service. When it is, this service is usually based in dermatology departments - another good reason to seek a dermatological opinion.

If hirsutism is mild and limited in extent, hair removal by shaving or depilatory creams may suffice. An alternative for treatment of facial hirsutism is eflornithine cream (Vaniqa $\left.{ }^{\circledR}\right){ }^{25}$ This is not a depilatory agent but 
Table 3 Treatment of hirsutism

Hair removal

- Depilatory creams, shaving, electrolysis, laser

Topical inhibition of hair growth

- Eflornithine

Suppression of androgen secretion and/or action

- Oral contraceptives

- Anti-androgens: cyproterone acetate (including co-cyprindiol); spironolactone; (flutamide)

- $5 \alpha$-reductase inhibitors: finasteride

rather an inhibitor of ornithine decarboxylase, an enzyme in the hair follicle that facilitates hair growth. It may take up to 3 months of use to demonstrate significant reduction in hair growth and treatment should be discontinued after 4 months if there is no noticeable improvement. Eflornithine cream is probably most effective when combined with laser treatment. ${ }^{26}$

In the medical management of hirsutism, combined oral contraceptives (COCs) play an important part by suppressing ovarian androgen production and therefore lowering serum testosterone levels. For women with mild hirsutism who also require contraception, the choice of more 'oestrogen-dominant' preparations such as Mercilon ${ }^{\circledR}$ or Marvelon ${ }^{\circledR}$ may be helpful, but in more significant hirsutism, greater benefit can be achieved by the use of an androgen receptor inhibitor such as cyproterone acetate or spironolactone. ${ }^{27} 28$ Yasmin $^{\circledR}$, a COC containing drospirenone, a spironolactone analogue, has also been advocated for use in hirsutism, but in common with other COCs it is not licensed for this purpose. Co-cyprindiol (initially marketed as Dianette ${ }^{\circledR}$ ) is a combined hormonal preparation containing cyproterone acetate $(2 \mathrm{mg})$ and is licensed for the treatment of severe acne as well as moderately severe hirsutism. There has been some concern about its long-term use, but the data suggesting that co-cyprindiol is associated with a greater risk of venous thromboembolism than third-generation 'pills' remain controversial and, in practice, ${ }^{29}$ it is a useful, usually well tolerated and effective treatment for hirsutism, with the additional (but unlicensed) major benefit of providing highly effective contraception.

Flutamide is a specific anti-androgen but is not recommended for treatment of hirsutism because of potential hepatotoxicity. It should not be prescribed in general practice. ${ }^{30}$ Finasteride is an inhibitor of $5 \alpha$-reductase, but its efficacy in treating hirsutism is difficult to evaluate because of a lack of appropriately powered randomised trials. ${ }^{31}$ Metformin has been claimed to reduce hair growth, but systematic analysis of the results of available studies points to only a small and insignificant effect. ${ }^{32}{ }^{33}$ Finally, it should be emphasised that suppression of ovarian function by long-acting analogues of gonadotrophin-releasing hormone ( $\mathrm{GnRH}$ agonists) is, in the great majority of cases, ineffective and unnecessary, as is oophorectomy. The exceptions are those rare cases where hirsutism results from hyperthecosis or androgen-secreting ovarian tumours. Postmenopausal exacerbation of hirsutism (or de novo onset) is an uncommon problem and requires investigation including measurement of testosterone, and imaging of the adrenals and ovaries to exclude tumours if testosterone levels exceed 5 $\mathrm{nmol} / \mathrm{l}$. In hyperthecosis, testosterone secretion remains LH-dependent, so suppression of LH with long-acting agonist analogues of GnRH will lower testosterone levels. This is useful both in diagnosis and management, although alternatives for treatment are anti-androgens and, for androgen-secreting tumours or for hyperthecosis after the menopause, oophorectomy.

The psychological impact of hirsutism can be considerable ${ }^{6}$ and a sympathetic approach to management is important, even in women whose hirsutism does not appear extensive or severe. In some cases, the resultant anxiety and depression may require psychotherapy or medication.

In summary, hirsutism is a very common and distressing problem. Its most common cause is PCOS, but a carefully obtained history will provide indicators of rare but more serious causes such as androgen-secreting tumours. Investigations should be targeted to the clinical presentation. Optimum management requires a combination of physical hair removal, hormonal treatments and, where necessary, psychological support.

Competing interests None.

Provenance and peer review Commissioned; externally peer reviewed.

\section{References}

1 Ferriman D, Gallwey JD. Clinical assessment of body hair growth in women. J Clin Endocrinol Metab 1961;21:1440-1447.

2 Koulouri O, Conway GS. Management of hirsutism. BMJ 2009;338:b847.

3 Yildiz BO, Bolour S, Woods K, et al. Visually scoring hirsutism. Hum Reprod Update 2010;16:51-64.

4 Barth JH, Catalan J, Cherry CA, et al. Psychological morbidity in women referred for treatment of hirsutism. J Psychosom Res 1993;37:615-619.

5 Archer JS, Chang RJ. Hirsutism and acne in polycystic ovary syndrome. Best Pract Res Clin Obstet Gynaecol 2004;18: 737-754.

6 Jones GL, Hall JM, Balen AH, et al. Health-related quality of life measurement in women with polycystic ovary syndrome: a systematic review. Hum Reprod Update 2008;14:15-25.

7 Kirschner MA, Bardin CW. Androgen production and metabolism in normal and virilized women. Metab Clin Exp 1972;21:667-688.

8 Gilling-Smith C, Franks S. Hirsutism and virilization. In Gynaecology (3rd edn), Shaw RW, Soutter WP, Stanton SL (eds). London, UK: Churchill Livingstone, 2003;387-399.

9 Pardridge WM. Serum bioavailability of sex steroid hormones. Clin Endocrinol Metab 1986;15:259-278.

10 Randall VA. Androgens and hair growth. Dermatol Ther 2008; 21:314-328. 
11 Hoffmann R. Enzymology of the hair follicle. Eur J Dermatol 2001;11:296-300.

12 Adams J, Polson DW, Franks S. Prevalence of polycystic ovaries in women with anovulation and idiopathic hirsutism. $\mathrm{Br} \mathrm{Med} \mathrm{J}$ (Clin Res Ed) 1986;293:355-359.

13 Franks S. Polycystic ovary syndrome. N Engl J Med 1995;333: 853-861.

14 Azziz R, Sanchez LA, Knochenhauer ES, et al. Androgen excess in women: experience with over 1000 consecutive patients. J Clin Endocrinol Metab 2004;89:453-462.

15 Ehrmann DA. Polycystic ovary syndrome. N Engl J Med 2005;352:1223-1236.

16 Kiddy DS, Sharp PS, White DM, et al. Differences in clinical and endocrine features between obese and non-obese subjects with polycystic ovary syndrome: an analysis of 263 consecutive cases. Clin Endocrinol (Oxf) 1990;32:213-220.

17 Barber TM, McCarthy MI, Wass JA, et al. Obesity and polycystic ovary syndrome. Clin Endocrinol (Oxf) 2006;65:137-145.

18 Pasquali R, Antenucci D, Casimirri F, et al. Clinical and hormonal characteristics of obese amenorrheic hyperandrogenic women before and after weight loss. J Clin Endocrinol Metab $1989 ; 68: 173-179$.

19 Kiddy DS, Hamilton-Fairley D, Bush A, et al. Improvement in endocrine and ovarian function during dietary treatment of obese women with polycystic ovary syndrome. Clin Endocrinol (Oxf) 1992;36:105-111.

20 Franks S, Gilling-Smith C, Watson $\mathrm{H}$, et al. Insulin action in the normal and polycystic ovary. Endocrinol Metab Clin North Am 1999;28:361-378.

21 Plymate SR, Matej LA, Jones RE, et al. Inhibition of sex hormone-binding globulin production in the human hepatoma (Hep G2) cell line by insulin and prolactin. J Clin Endocrinol Metab 1988;67:460-464.

22 Bidet M, Bellanné-Chantelot C, Galand-Portier MB, et al. Clinical and molecular characterization of a cohort of 161 unrelated women with nonclassical congenital adrenal hyperplasia due to 21-hydroxylase deficiency and 330 family members. J Clin Endocrinol Metab 2009;94:1570-1578.

23 Rosner W, Vesper H. Toward excellence in testosterone testing: a consensus statement. J Clin Endocrinol Metab 2010;95:4542-4548.

24 Koulouri O, Conway GS. A systematic review of commonly used medical treatments for hirsutism in women. Clin Endocrinol (Oxf) 2008;68:800-805.

25 Wolf JE Jr, Shander D, Huber F, et al. Randomized, doubleblind clinical evaluation of the efficacy and safety of topical eflornithine $\mathrm{HCl} 13.9 \%$ cream in the treatment of women with facial hair. Int J Dermatol 2007;46:94-98.

26 Lapidoth M, Dierickx C, Lanigan S, et al. Best practice options for hair removal in patients with unwanted facial hair using combination therapy with laser: guidelines drawn up by an expert working group. Dermatology (Basel) 2010;221:34-42.

27 Barth JH, Cherry CA, Wojnarowska F, et al. Spironolactone is an effective and well tolerated systemic antiandrogen therapy for hirsute women. J Clin Endocrinol Metab 1989;68:966-970.

28 Barth JH, Cherry CA, Wojnarowska F, et al. Cyproterone acetate for severe hirsutism: results of a double-blind dose-ranging study. Clin Endocrinol (Oxf) 1991;35:5-10.

29 Franks S, Layton A, Glasier A. Cyproterone acetate/ethinyl estradiol for acne and hirsutism: time to revise prescribing policy. Hum Reprod 2008;23:231-232.

30 Castelo-Branco C, Moyano D, Gómez O, et al. Long-term safety and tolerability of flutamide for the treatment of hirsutism. Fertil Steril 2009;91:1183-1188.

31 Swiglo BA, Cosma M, Flynn DN, et al. Clinical review: antiandrogens for the treatment of hirsutism: a systematic review and metaanalyses of randomized controlled trials. J Clin Endocrinol Metab 2008;93:1153-1160.

32 Costello MF, Shrestha B, Eden J, et al. Metformin versus oral contraceptive pill in polycystic ovary syndrome: a Cochrane review. Hum Reprod 2007;22:1200-1209.

33 Franks S. When should an insulin sensitizing agent be used in the treatment of polycystic ovary syndrome? Clin Endocrinol (Oxf) 2011;74:148-151. 